\title{
Biexciton Formation in Bilayer Tungsten Disulfide
}

Zhengyu He ${ }^{l}$, Wenshuo Xu ${ }^{l}$, Yingqiu Zhou ${ }^{l}$, Xiaochen $\mathrm{Wang}^{1}$, Yuewen Sheng ${ }^{l}$, Youmin Rong $^{1}$, Shaoqiang Guo ${ }^{2}$, Junying Zhang ${ }^{2}$, Jason M. Smith ${ }^{1}$, Jamie H. Warner ${ }^{\text {* }}$

${ }^{1}$ Department of Materials, University of Oxford, Parks Road, Oxford, OX1 3PH, United Kingdom

${ }^{2}$ Department of Physics, Beihang University, Beijing 100191, People's Republic of China

*Jamie.warner@materials.ox.ac.uk;

\begin{abstract}
.
Monolayer transition metal dichalcogenides (TMDs) are direct band gap semiconductors and their 2D structure results in large binding energies for excitons, trions and biexcitons. The ability to explore many-body effects in these monolayered structures has made them appealing for future opto-electronic and photonic applications. The band structure changes for bilayer TMDs with increased contributions from indirect transitions and this has limited similar indepth studies of biexcitons. Here, we study biexciton emission in bilayer $\mathrm{WS}_{2}$ grown by chemical vapour deposition as a function of temperature. A biexciton binding energy of $36+/-$ $4 \mathrm{meV}$ is measured in the as grown bilayer $\mathrm{WS}_{2}$ containing $0.4 \%$ biaxial strain as determined by Raman spectroscopy. The biexciton emission was difficult to detect when the $\mathrm{WS}_{2}$ was transferred to another substrate to release the stain. Density functional theory (DFT) calculations show that $0.4 \%$ of tensile strain lowers the direct bandgap by about $55 \mathrm{meV}$ without significant change to the indirect bandgap, which can cause an increase in the quantum yield of direct exciton transitions and the emission from biexcitons formed by two direct gap excitons. We find that the biexciton emission decreases dramatically with increased temperature due to the thermal dissociation, with an activation energy of $26+/-5 \mathrm{meV}$. These
\end{abstract}


results show how strain can be used to tune the many-body effects in bilayered TMD materials and extend the photonic applications beyond pure monolayer systems.

KEYWORDS Transition metal dichalcogenide, Tungsten Disulfide, biexciton, Photoluminescence.

Transition metal dichalcogenides (TMDCs) are layered materials with buckled hexagonal lattice, composed of three atomic layers with transition metal atoms sandwiched by two layers of chalcogenide atoms. Monolayer TMDCs are direct-gap semiconductors with broken inversion symmetry, exhibiting giant excitonic effects ${ }^{1-6}$ as well as valley physics. ${ }^{7-12}$ The combination of two monolayers through van der Waals forces causes the direct- to indirect-gap crossover from monolayer to bilayer TMDCs due to interlayer coupling ${ }^{13-17}$, and the detailed band structure depends on the twist angle between the two lattices, providing means to manipulate the properties of TMDCs. Huang et al. ${ }^{6}$ reported bilayer molybdenum disulfide $\left(\mathrm{MoS}_{2}\right)$ with different twisted angles can tune the trion binding energy and therefore the photoluminescence (PL) emission. Bilayer tungsten disulfide $\left(\mathrm{WS}_{2}\right)$ also possesses opposite optical response towards an in-plane electric field compared with a monolayer crystal. ${ }^{18}$ Unlike its monolayer counterpart, valley physics is expected to fade away in bilayer TMDCs due to the recovery of inversion symmetry. However, $\mathrm{Wu}$ et al. ${ }^{19}$ proved that the symmetry is electrically controllable, resulting in the realization of continuous and reversible manipulation of valley-contrasting optical properties. Furthermore, Zhu et al. ${ }^{20}$ showed that bilayer $\mathrm{WS}_{2}$ exhibits even more pronounced valley polarization and valley coherence compared with those 
in monolayer $\mathrm{WS}_{2}$, thanks to the much stronger valley-spin coupling (about $0.4 \mathrm{eV}$ ) than the interlayer hopping energy $(0.1 \mathrm{eV}) .^{20}$

Apart from excitonic ground states, atomically thin TMDCs offer an excellent platform to explore fascinating higher-order many-body effects ${ }^{9}$, e.g., biexcitons which are rarely accessible to in bulk materials due to the enhanced dielectric screening and lack of confinement. For example, Sie et al. ${ }^{21}$ claimed the observation of both homo- and hetero-biexciton in the transient absorption measurement on monolayer $\mathrm{MoS}_{2}$. You et al. ${ }^{22}$ and Shang et al. ${ }^{23}$ reported the creation of bright biexcitons within monolayer $\mathrm{WS}_{2}$ and $\mathrm{WSe}_{2}$ by injecting dense excitons which can subsequently condense to the biexciton by emission of acoustic phonons. ${ }^{24}$ Being able to create a biexciton or even multiple exciton states will help understand prominent manybody effects within TMDCs. Additionally, biexcitons can be utilized to develop many practical applications including single photon sources, ${ }^{25,26}$ and lasers. ${ }^{27,28}$

Here we present our observation of biexciton formation within as-grown CVD bilayer $\mathrm{WS}_{2}$ by creating a dense exciton gas under a cryogenic environment (77 K). Previous reports have claimed that biexcitons in indirect-gap semiconductors have been conclusively identified because of the relatively long life time of the indirect gap exciton. ${ }^{29-31}$ However, it should be noted that the biexcitons here are associated with direct-gap excitons, the quantum yield of which is much smaller than that in direct-gap monolayer $\mathrm{WS}_{2}$. Yet this high-order excitonic emission was absent in transferred bilayer $\mathrm{WS}_{2}$ due to strain release during the transfer process. Temperature dependent measurements showed that the biexciton emission strength decreases 
dramatically with increasing temperature, which is the result of increasing thermal dissociation. ${ }^{23}$

\section{Results and Discussion}

Atomically thin tungsten disulfide crystals were synthesized by chemical vapour deposition (CVD) using a previously reported method. ${ }^{32}$ Careful selection of parameters can yield primarily monolayer crystals, but also domains where monolayer and few-layer $\mathrm{WS}_{2}$ coexist, as shown in figure 1a. Optimization of the RGB channel of the imaging CCD can help distinguish monolayer, bilayer and few-layer region by optical contrast. ${ }^{33}$ PL spectra confirmed that the darker region shown in the inset in figure $1 \mathrm{~b}$ was more than one atomic layer with much weaker photoluminescence (PL) intensity (red curve, figure 1b) than that of monolayer region (black curve, figure $1 \mathrm{~b}$ ) due to the indirect bandgap of multilayer $\mathrm{WS}_{2} \cdot{ }^{14,34}$ To further prove that the darker region was bilayer, we performed atomic force microscopy on the region highlighted in blue rectangle including the boundary between regions with two different optical contrast in the inset in figure $1 \mathrm{~b}$ and the topography was presented in figure $1 \mathrm{c}$. It clearly showed that there was a step at the boundary of regions with different optical contrast, with a height difference of about $0.7 \mathrm{~nm}$, indicating that there were two atomic layers in the darker region. Raman signatures can also provide insight into the determination of number of layers. Figure 1d and 1e showed the Raman spectra of the monolayer and bilayer with excitation wavelength $\lambda_{\text {exc }}=532 \mathrm{~nm}$. Multi-Lorentzian curve fitting was performed, from which we extracted peak positions in the monolayer for $\mathrm{E}^{\prime}(\Gamma)$ and $\mathrm{A}^{\prime}{ }_{1}(\Gamma)$ of $356.8 \mathrm{~cm}^{-1}$ and $418.6 \mathrm{~cm}^{-}$

${ }^{1}$ respectively. In the bilayer, the $\mathrm{E}_{\mathrm{g}}{ }^{1}(\Gamma)$ mode was softened, with its peak at $354.9 \mathrm{~cm}^{-1}$, and the $\mathrm{A}_{\lg }(\Gamma)$ mode experienced stiffening with a peak position of $420.0 \mathrm{~cm}^{-1}$. These values are consistent with those for bilayer $\mathrm{WS}_{2}$ reported in other literature. ${ }^{35}$ The $2 \mathrm{LA}(\mathrm{M})$ peak was more pronounced than $E^{\prime}\left(E_{2 g}{ }^{1}\right)$ in monolayer (bilayer) under the $532 \mathrm{~nm}$ laser excitation due to 
double resonance. ${ }^{36}$ Description of other Raman signatures can be found in the supplementary information. The notations $E^{\prime}\left(E_{g}{ }^{1}\right)(\Gamma)$ and $A^{\prime}{ }_{1}\left(A_{1 g}\right)(\Gamma)$ for monolayer (bilayer) correspond to vibration modes which were denoted as $\mathrm{E}_{2 \mathrm{~g}}{ }^{1}(\Gamma)$ and $\mathrm{A}_{\lg }(\Gamma)$ in previous literature. ${ }^{37}$ The difference in notations comes from the symmetry difference between bulk and monolayer or bilayer which were discussed in details in previous report. ${ }^{38}$
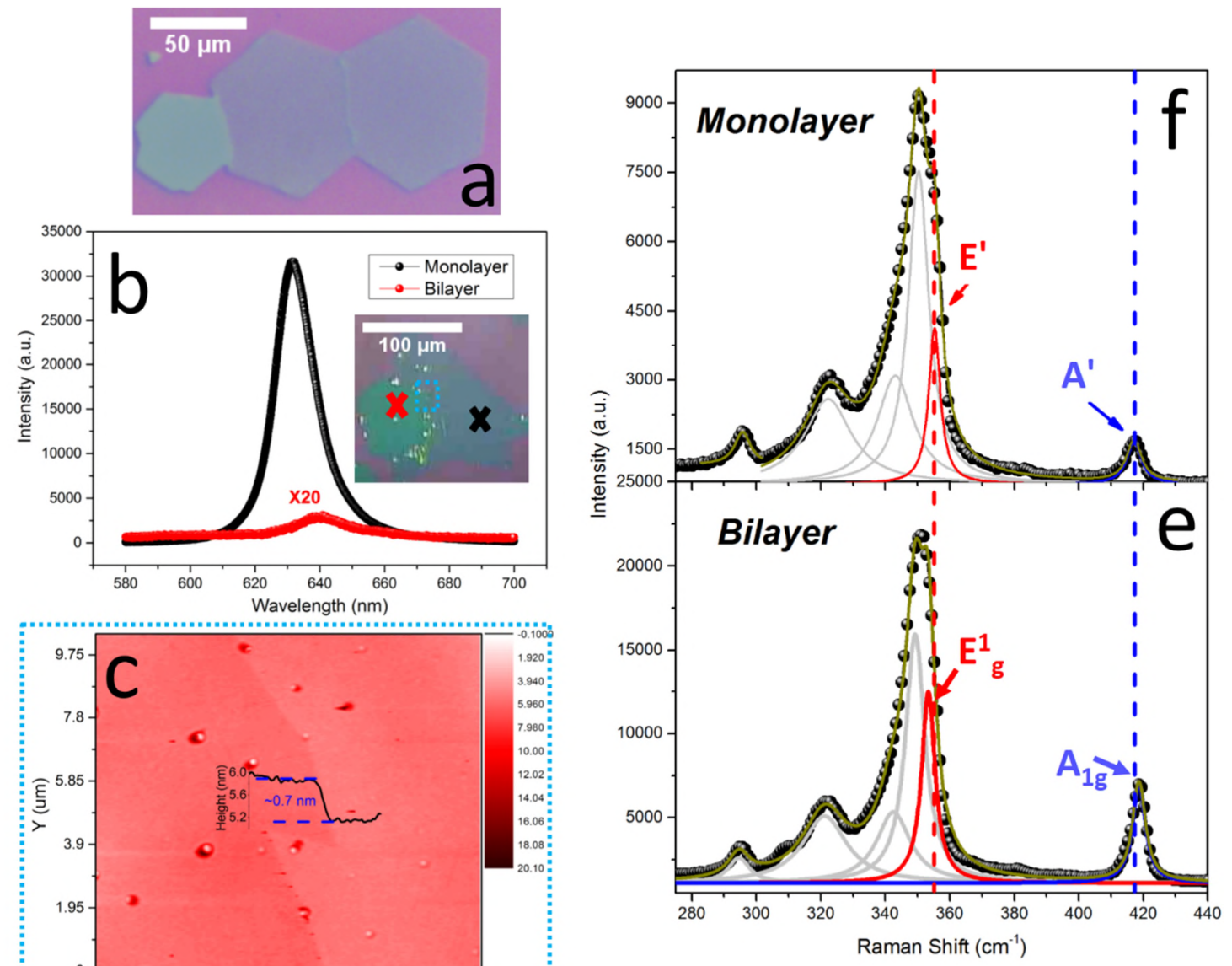

Figure 1. (a) Optical image of $\mathrm{WS}_{2}$ domain with both monolayer and few-layer coexisting. (b) Photoluminescence spectra of monolayer (black) from region marked by black cross in the inset and bilayer (red) from area marked by red cross. Inset: optical image of $\mathrm{WS}_{2}$ domain with both monolayer and bilayer. (c) AFM image of $\mathrm{WS}_{2}$ highlighted by red rectangle in the inset of panel (a). (d,e) Raman spectra of (d) monolayer and (e) bilayer. Red curve: fitting results of $E^{\prime}\left(E_{g}{ }^{1}\right)$. Blue curve: fitting results of A'( $\left.A_{1 g}\right)$. Light grey curve: other Raman vibration modes. 
The excitation power dependence of the PL intensity can provide insights into the physical origin of the corresponding PL peaks. ${ }^{39}$ High power excitation can also lead to the emergence of new PL peaks originating from high-order excitonic states, providing insights into the manybody effect within the materials. ${ }^{22,23}$ Figure 2(a)-(c) depict three PL spectra of bilayer $\mathrm{WS}_{2}$ at liquid nitrogen $(\mathrm{LN})$ temperature $(77 \mathrm{~K})$ under different excitation power. Under low excitation power (figure 2(a)), the PL spectrum is deconvoluted by only four peaks, which are assigned to exciton (X, red curve), trion ( $\mathrm{X}^{-}$, green curve), localized states (LS, blue curve) and indirect transition (I, light blue curve). The LS peak probably originates from a disorder effect ${ }^{40}$ and impurities. ${ }^{22}$ With increasing laser power, a new peak gradually emerges (figure 2(b) and 2(c), dark yellow curve). In order to determine the physical origin of this new peak, power dependence of integrated PL intensity of exciton, trion and the new peak are extracted and depicted in figure 2(e). Emission from X increases almost linearly with excitation power while integrated PL intensity of the trion possesses a slightly superlinear dependence with power law exponent of 1.2. The superlinearity of the trion emission has been reported before and may be due to the loss of trion population to LS at low density ${ }^{23}$. In contrast, the integrated emission intensity of the emerging peak exhibits a superlinear relationship with laser power, with a power law exponent of $\sim 1.6$. We further depict the emission intensity of the emerging peak as a function of integrated exciton PL intensity in figure 2(f), showing a nearly quadratic relationship (power law exponent of $\sim 1.9$ ). This provides strong evidence that the new peak is associated with biexcitons. It should be pointed out that the quadratic relationship between emission intensity of biexcitons and the excitation power is not always satisfied. More direct evidence is the quadratic relationship between the emission strength of biexcitons relative to the excitons, as presented in figure 2(f) (see supplementary information for more discussion). ${ }^{23,24}$ Figure $2(\mathrm{~g}),(\mathrm{h})$ shows the peak positions and full-width-half-maximum (FWHM) of $\mathrm{X}, \mathrm{X}^{-}$and $\mathrm{X}_{\mathrm{A}} \mathrm{X}_{\mathrm{A}}$, from which we extract the binding energy for a trion of about 20 
meV, smaller than what has been reported in the literature. ${ }^{20}$ However, it should be noted that a difference in trion binding energy has been reported between CVD as-grown and mechanically exfoliated monolayer $\mathrm{TMDs}^{22}$, which is possibly due to the different doping levels ${ }^{34}$ and strain within the as-grown sample. The binding energy of biexcitons is measured to be about $36+/-4 \mathrm{meV}$, smaller than that in monolayer TMDs due to the reduced exciton binding energy in bilayer $\mathrm{WS}_{2}$. The exciton binding energy has been reported to be $0.36 \mathrm{eV} \sim$ $0.7 \mathrm{eV}^{2,41,42}$ in monolayer $\mathrm{WS}_{2}$ while the binding energy in the bilayer material is about half of that ${ }^{33}$ Comparing the exciton and biexciton binding energies, we can extract a ratio called the Haynes factor ${ }^{43}$ which is about $0.09 \sim 0.2$, similar to what has been reported in monolayer TMDs. ${ }^{22,23}$ 

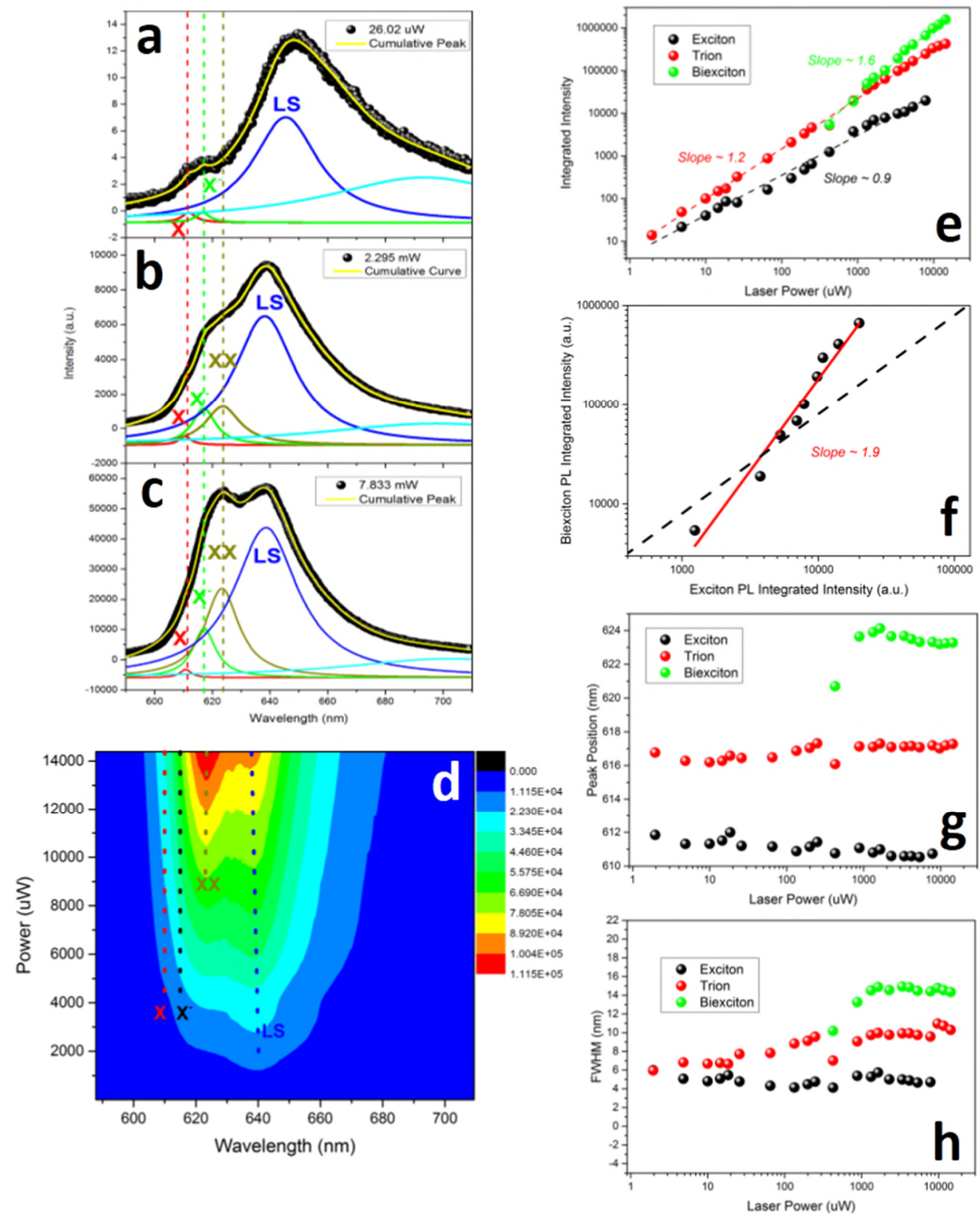

Figure 2. Power dependent PL of bilayer $\mathrm{WS}_{2}$ at $77 \mathrm{~K}$. (a-c) PL spectra of bilayer $\mathrm{WS}_{2}$ under different laser excitation powers. Dashed lines in a-c are guides to show peak positions. (d) Power dependent PL color contour. (e) Logarithmic plot of the PL integrated intensity of the exciton, trion and biexciton peaks versus laser excitation power. (f) Logarithmic plot of the biexciton integrated intensity versus exciton integrated intensity. The dashed line has a slope of 1 for comparison. (g) Semilogarithmic plot of PL peak positions of exciton, trion and biexciton versus laser excitation power. (h) Semilogarithmic plot of FWHM varitions of excitons, trions and biexcitons versus excitation power. 
We also performed power dependent PL on transferred bilayer $\mathrm{WS}_{2}$ shown in figure 3(a)-(c), where no obvious biexciton emission was observed. Under low excitation power $\left(\sim 10^{2} \mathrm{~W} / \mathrm{cm}^{2}\right)$, the spectrum is dominated by LS states emission while under high power $\left(\sim 10^{2} \mathrm{~kW} / \mathrm{cm}^{2}\right)$, exciton and trion emission become more pronounced. Figure 3(b) shows the Raman spectra of both as-grown and transferred bilayer $\mathrm{WS}_{2}$, which can be used to analyze the doping level as well as lattice strain. ${ }^{44,45}$ The $\mathrm{A}_{1 \mathrm{~g}}(\ulcorner)$ mode is related to the out-of-plane vibration of sulphur atoms and therefore is sensitive to the doping level. In our experiment, the peak position of $\mathrm{A}_{1 \mathrm{~g}}$ mode changes little after the transfer process (blue curve in figure 3(b)), indicating that the doping level of our bilayer $\mathrm{WS}_{2}$ remains almost unchanged. The $\mathrm{E}_{\mathrm{g}}{ }^{1}(\Gamma)$ mode is associated with the in-plane vibration of tungsten and sulphur atoms, which is sensitive to the change of lattice strain. As shown in figure 3(b), the $\mathrm{E}_{\mathrm{g}}{ }^{1}(\Gamma)$ mode is softened by about $1.3 \mathrm{~cm}^{-1}$ (red curve in figure 3(b)) on the transferred sample, indicating the release of lattice strain, which is consistent with other literature ${ }^{46}$. The Gruneisen parameter can link the lattice strain with the $\mathrm{Eg}_{\mathrm{g}}{ }^{1}$ phonon mode shift quantitatively, obeying the following relationship: $\gamma_{\mathrm{G}}=-\Delta \omega_{G} / 2 \omega_{G}^{0} \epsilon$, where $\gamma_{\mathrm{G}}$ is the Gruneisen parameter for mode, $\Delta \omega_{G}$ is the peak shift after strain is applied, $\omega_{G}^{0}$ is the Raman peak position with zero strain and $\epsilon$ is the strain applied. For monolayer $\mathrm{WS}_{2}$, the Gruneisen parameter is $0.54^{47}$ while for bilayer and few-layer $\mathrm{WS}_{2}$ this parameter changes little $^{48}$. Therefore, the as-grown bilayer $\mathrm{WS}_{2}$ possessed a typical biaxial tensile strain of $\sim 0.4 \%$ (see supplementary information for detailed analysis), which comes from the difference in thermal expansion coefficient between the $\mathrm{WS}_{2}$ and the substrate. Given that strain has been linked to indirect-to-direct transition crossover in multilayer $\mathrm{WSe}_{2},{ }^{49}$ one plausible explanation to the absence of the biexciton emission from transferred bilayer $\mathrm{WS}_{2}$ is that the release of strain during the transfer process would degrade the quantum yield of the direct transition within bilayer $\mathrm{WS}_{2}$. In figure 3(f), we presented the DFT calculated band structure of both strain-free (black) and $0.4 \%$ strained (red) bilayer $\mathrm{WS}_{2}$. It shows that strain could induce a 
measureable downshift of the conduction band valley at the K point (labelled by blue circle in figure 3(f)) while other parts of the band structure remained almost unchanged. The band gap values of both direct transition and indirect transition with and without strain were extracted in figure $3(\mathrm{~g})$, which clearly show that $0.4 \%$ strain could result in the reduction of direct transition band gap by $55 \mathrm{meV}$ while the indirect band gap changes little. This can lead to the increase of quantum yield of direct transitions ${ }^{49}$ and therefore the increase of biexciton emission. A similar study has also been carried out on as-grown multilayer $\mathrm{WS}_{2}$ but no biexciton was observed even under the highest excitation power we can reach, which was probably as a result of the reduced binding energy as well as low quantum yield of direct recombination. 

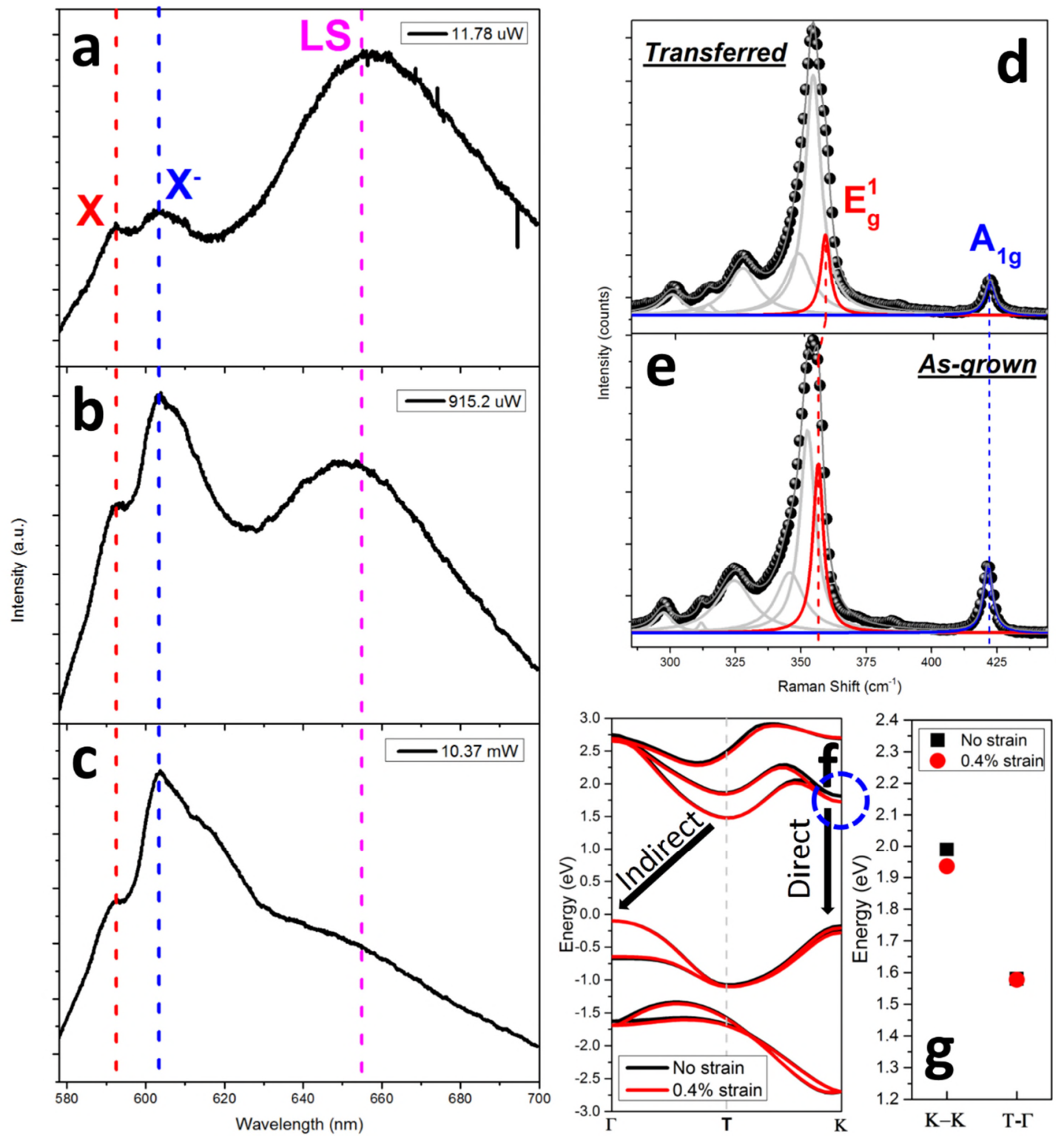

Figure 3. (a-c) PL spectra of transferred bilayer $\mathrm{WS}_{2}$ under different excitation power. (d,e) Raman spectra of both (d) transferred and (e) As-grown layer $\mathrm{WS}_{2}$. Red: fitting curve for $\mathrm{E}_{\mathrm{g}}{ }^{1}$. Blue: fitting curve for $A_{1 g}$. (f) DFT calculated band structures of strainless (black curve) and strained (red curve). (g) Band gap of direct $\mathrm{K}-\mathrm{K}$ direct transition and $\mathrm{T}-\Gamma$ indirect transition of bilayer $\mathrm{WS}_{2}$ with (red circles) and without strain (black squares).

We also performed power dependent PL measurements on as-grown bilayer $\mathrm{WS}_{2}$ at room temperature. In figure 4(a), we show a typical PL spectra taken at room temperature under three different laser excitation power. Two characteristic PL peaks can be distinguished in the spectra, and are attributed to the direct (X) and indirect gap (I) transitions, respectively. No obvious 
peak shape change was found under high excitation power, indicating that no new excitonic species were created. Figure 4(b) shows that the excitation power dependence of $\mathrm{X}$ emission is highly linear, which is the typical power dependence for excitonic ground state emission. We have also investigated the variation of the peak position and FWHM with excitation laser power, shown in figure 4(c). Although no change in the peak shape was observed under high power excitation, the $\mathrm{X}$ peak experienced a redshift and broadening, likely due to laser induced heating. ${ }^{35}$ In figure $4(\mathrm{~d})$, we present the temperature dependence of the biexciton emission intensity under fixed excitation power to investigate the thermal stability of the biexcitons. (See supplementary for raw PL data) With increasing temperature, the biexciton emission diminished greatly and was hardly resolved if the temperature exceeded $150 \mathrm{~K}$. Such behaviour can be explained by a model in which the formation rate of biexciton remains unchanged while the dissociation rate can be thermally activated. ${ }^{23}$ The temperature dependent decay rate of the biexciton population can be described in the form of $\gamma(\mathrm{T})=\tau_{X X}^{-1}+k_{0} \exp \left(E_{a} / k_{B} T\right)$, where $\tau_{X X}$ is the life time of the biexciton, $\mathrm{k}_{0}$ is a prefactor and $\mathrm{E}_{\mathrm{a}}$ is the dissociation energy. ${ }^{23}$ The biexciton intensity is proportional to $\gamma^{-1}(\mathrm{~T})$. In figure $4 \mathrm{f}$ the red curve represented the best fit to the data, yielding a dissociation energy of about $26+/-5 \mathrm{meV}$. This value is smaller than the binding energy of biexcitons, indicating that biexciton may dissociate into excitonic species other than two bright excitons with increasing temperature. 

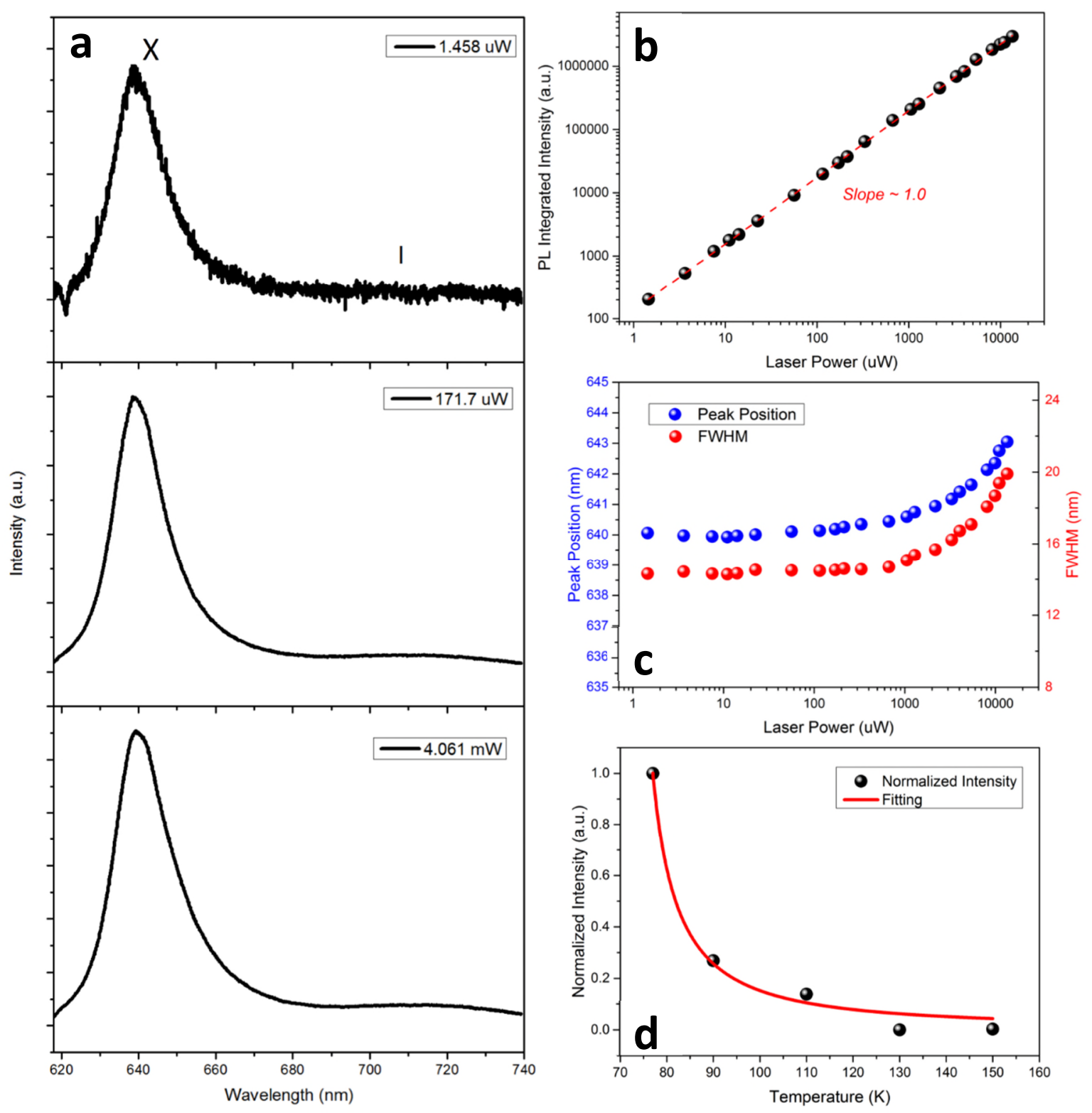

Figure 4. PL of bilayer $\mathrm{WS}_{2}$. (a) Typical PL spectra of bilayer $\mathrm{WS}_{2}$ at room temperature under three different laser excitation power. (b) Logarithmic plot of the PL integrated intensity of exciton A versus laser excitation power. Red line: linear fitting line, with slope of about 1.0. (c) Semilogarithmic plot of the exciton peak position and full-width-half-maximum (FWHM) variation versus laser excitation power. (d) Temperature dependence of the biexciton emission intensity. Red curve: fitting result according to the temperature dependent biexciton population decay rate described in the main text.

\section{Conclusion}


In summary, we observed biexciton emission from as-grown CVD bilayer $\mathrm{WS}_{2}$ under high power excitation with a binding energy of $36 \mathrm{meV}$, corresponding to a Haynes factor within the range of $0.09 \sim 0.20$. However, such high-order excitonic emission was not detected in transferred samples. Raman spectroscopy revealed that biaxial strain of about $0.4 \%$ was present within the as-grown sample, resulting in an increased quantum yield of direct recombination which facilitated biexciton formation. We have also investigated the thermal stability of biexciton emission, which is suppressed dramatically with increased temperature due to the increasing thermal dissociation with dissociation energy of about $26 \mathrm{meV}$.

\section{Methods}

Synthesis and Transfer of $W S_{2}$ :

$\mathrm{WS}_{2}$ bilayer domains are prepared using our previously reported CVD method with sulphur and $\mathrm{WO}_{3}$ as the precursor. Sulphur and $\mathrm{WO}_{3}$ are placed in a 1 inch quartz tube running through two furnace systems to provide two heating sections. Vaporized sulphur and $\mathrm{WO}_{3}$ are carried by flowing argon gas to the reaction zone, where $\mathrm{WO}_{3}$ undergoes sulphurization. High quality and large area $\mathrm{WS}_{2}$ domains with atomic layer thickness are grown on Si wafers with $300 \mathrm{~nm}$ $\mathrm{SiO}_{2}$ if proper parameters including temperature, Ar flow rate, sulphur introduction time are achieved.

During the transfer process, a thin layer of PMMA (495 K, A8, MicroChem) was spin coated onto the as-grown sample, followed by an etching process in which PMMA coated samples floated on $1 \mathrm{~mol} / \mathrm{L} \mathrm{KOH} \mathrm{(Sigma} \mathrm{Aldrich)} \mathrm{solution} \mathrm{immersed} \mathrm{water} \mathrm{bath} \mathrm{at} 45^{\circ} \mathrm{C}$ for about $1 \mathrm{hr}$. The detached film was then transferred to target substrate. In this case, the target substrate was $\mathrm{SiO}_{2} / \mathrm{Si}$ wafer.

PL Measurement: 
In the photoluminescence (PL) measurement, a $532 \mathrm{~nm}$ diode-pumped solid state laser (Thorlabs, DJ532-40) was used for excitation. The laser was reflected off a dichroic beam splitter and focused to a spot size of $\sim 2 \mu \mathrm{m}$ by $50 \times$ ultra-long working distance objective. $\mathrm{WS}_{2}$ was placed in a flow microscopy cryostat (Janis, ST-500) with continuous liquid nitrogen flowing to keep the temperature constant at $77 \mathrm{~K}$ under vacuum of $\sim 1 \times 10^{-6} \mathrm{mbar}$. PL spectra were collected by a custom-built confocal microscope imaging system coupled to a spectrometer with an attached CCD (Princeton Instruments Acton SP-2300 spectrometer with Princeton Instruments, PIXIS 100 CCD).

Aberration Corrected Transmission Electron Microscopy (AC-TEM)

AC-TEM was performed using Oxford's JEOL 2200MCO, with a CEOS image corrected and accelerating voltage of $80 \mathrm{kV}$. Image analysis was performed using ImageJ.

\section{Band Structure Calculation:}

DFT calculations were performed using Vienna ab initio simulation package $\left(\right.$ VASP) ${ }^{50}$ with electronic exchange-correlation energy described by Perdew-Burke-Ernzerhof $(\mathrm{PBE})^{51}$ function of generalized gradient approximation (GGA). The basis set contains plane waves with cut-off energy up to $450 \mathrm{eV}$. The Brillouin zone (BZ) was sampled with Monkhorst-Pack $k$-points mesh of $9 \times 9 \times 1$. Bilayer $\mathrm{WS}_{2}$ was modelled in a slab geometry by containing a vacuum region of $15 \AA$ along z-axis. For strained bilayer $\mathrm{WS}_{2}$, structure was first fully relaxed until the total energy was converged to $10^{-4} \mathrm{eV}$ and the force on each atom was smaller 0.01 $\mathrm{eV} / \mathrm{A}$ to determine the optimized lattice constants.

Supporting Information Available: 
Additional information about more evidence on biexciton formation in non-transferred bilayer $\mathrm{WS}_{2}$, detailed Raman analysis, peak fitting parameters and temperature dependent PL spectra. This materials is available free of charge via the Internet at http://pubs.acs.org.

\section{Acknowledgements}

JHW thanks the Royal Society for support. ZH thanks the CSC and the China-Oxford scholarship fund for support.

\section{References:}

(1) Ramasubramaniam, A. Large Excitonic Effects in Monolayers of Molybdenum and Tungsten Dichalcogenides. Phys. Rev. B 2012, 86, 115409.

(2) Zhu, B.; Chen, X.; Cui, X. Exciton Binding Energy of Monolayer WS2. Sci. Rep. 2014, 5, 9218.

(3) Splendiani, A.; Sun, L.; Zhang, Y.; Li, T.; Kim, J.; Chim, C.-Y.; Galli, G.; Wang, F. Emerging Photoluminescence in Monolayer MoS2. Nano Lett. 2010, 10, 1271-1275.

(4) Lopez-Sanchez, O.; Alarcon Llado, E.; Koman, V.; Fontcuberta i Morral, A.; Radenovic, A.; Kis, A. Light Generation and Harvesting in a Van Der Waals Heterostructure. ACS Nano 2014, $8,3042-3048$.

(5) Ross, J. S.; Klement, P.; Jones, A. M.; Ghimire, N. J.; Yan, J.; Mandrus, D. G.; Taniguchi, T.; Watanabe, K.; Kitamura, K.; Yao, W.; Cobden, D. H.; Xu, X. Electrically Tunable Excitonic Light-Emitting Diodes Based on Monolayer WSe2 P-N Junctions. Nat. Nanotechnol. 2014, 9, $268-272$.

(6) Huang, S.; Ling, X.; Liang, L.; Kong, J.; Terrones, H.; Meunier, V. Probing the Interlayer Coupling of Twisted Bilayer MoS2 Using Photoluminescence Spectroscopy. Nano Lett. 2014, 14, 5500-5508. 
(7) Jones, A. M.; Yu, H.; Ghimire, N. J.; Wu, S.; Aivazian, G.; Ross, J. S.; Zhao, B.; Yan, J.; Mandrus, D. G.; Xiao, D.; Yao, W.; Xu, X. Optical Generation of Excitonic Valley Coherence in Monolayer WSe2. Nat. Nanotechnol. 2013, 8, 634-638.

(8) Cao, T.; Wang, G.; Han, W.; Ye, H.; Zhu, C.; Shi, J.; Niu, Q.; Tan, P.; Wang, E.; Liu, B.; Feng, J. Valley-Selective Circular Dichroism of Monolayer Molybdenum Disulphide. Nat. Commun. 2012, 3, 887.

(9) Mai, C.; Barrette, A.; Yu, Y.; Semenov, Y. Many-Body Effects in Valleytronics: Direct Measurement of Valley Lifetimes in Single-Layer MoS2. Nano Lett. 2013, 14, $202-206$.

(10) Wu, S. F.; Ross, J. S.; Liu, G. B.; Aivazian, G.; Jones, A.; Fei, Z. Y.; Zhu, W. G.; Xiao, D.; Yao, W.; Cobden, D.; Xu, X. D. Electrical Tuning of Valley Magnetic Moment through Symmetry Control in Bilayer MoS2. Nat. Phys. 2013, 9, 149-153.

(11) Xiao, D.; Liu, G. Bin; Feng, W.; Xu, X.; Yao, W. Coupled Spin and Valley Physics in Monolayers of MoS 2 and Other Group-VI Dichalcogenides. Phys. Rev. Lett. 2012, 108, 196802.

(12) Zeng, H.; Liu, G.-B.; Dai, J.; Yan, Y.; Zhu, B.; He, R.; Xie, L.; Xu, S.; Chen, X.; Yao, W.; Cui, X. Optical Signature of Symmetry Variations and Spin-Valley Coupling in Atomically Thin Tungsten Dichalcogenides. Sci. Rep. 2013, 3, 1608.

(13) Fang, H.; Battaglia, C.; Carraro, C.; Nemsak, S.; Ozdol, B.; Kang, J. S.; Bechtel, H. a; Desai, S. B.; Kronast, F.; Unal, A. a; Conti, G.; Conlon, C.; Palsson, G. K.; Martin, M. C.; Minor, A. M.; Fadley, C. S.; Yablonovitch, E.; Maboudian, R.; Javey, A. Strong Interlayer Coupling in van Der Waals Heterostructures Built from Single-Layer Chalcogenides. Proc. Natl. Acad. Sci. U. S. A. 2014, 111, 6198-6202.

(14) Mak, K. F.; Lee, C.; Hone, J.; Shan, J.; Heinz, T. F. Atomically Thin MoS2: A New DirectGap Semiconductor. Phys. Rev. Lett. 2010, 105, 136805. 
(15) Peimyoo, N.; Shang, J.; Cong, C.; Shen, X.; Wu, X.; Yeow, E. K. L.; Yu, T. Nonblinking, Intense Two-Dimensional Light Emitter: Monolayer WS2 Triangles. ACS Nano 2013, 7, 10985-10994.

(16) Tongay, S.; Zhou, J.; Ataca, C.; Lo, K.; Matthews, T. S.; Li, J.; Grossman, J. C.; Wu, J. Thermally Driven Crossover from Indirect toward Direct Bandgap in 2D Semiconductors: MoSe2 versus MoS2. Nano Lett. 2012, 12, 5576-5580.

(17) Zhao, W.; Ribeiro, R. M.; Toh, M.; Carvalho, A.; Kloc, C.; Castro Neto, A. H.; Eda, G. Origin of Indirect Optical Transitions in Few-Layer MoS2, WS2, and WSe2. Nano Lett. 2013, 13, $5627-5634$.

(18) He, Z.; Sheng, Y.; Rong, Y.; Lee, G.; Li, J.; Warner, J. H. Layer-Dependent Modulation of Tungsten Disul Fi de Photoluminescence by Lateral Electric Fields. ACS Nano 2015, 9, 2740 2748.

(19) Wu, S. F.; Ross, J. S.; Liu, G. B.; Aivazian, G.; Jones, A.; Fei, Z. Y.; Zhu, W. G.; Xiao, D.; Yao, W.; Cobden, D.; Xu, X. D. Electrical Tuning of Valley Magnetic Moment through Symmetry Control in Bilayer MoS2. Nat. Phys. 2013, 9, 149-153.

(20) Zhu, B.; Zeng, H.; Dai, J.; Gong, Z.; Cui, X. Anomalously Robust Valley Polarization and Valley Coherence in Bilayer WS2. Proc. Natl. Acad. Sci. U.S.A 2014, 111, 11606-11611.

(21) Sie, E. J.; Lee, Y.; Frenzel, A. J.; Kong, J.; Gedik, N. Intervalley Biexcitons and Many-Body Effects in Monolayer MoS2. Phys. Rev. B - Condens. Matter Mater. Phys. 2015, 92, 125417.

(22) Shang, J.; Shen, X.; Cong, C.; Peimyoo, N.; Cao, B.; Eginligil, M. Observation of Excitonic Fine Structure in a 2D Transition-Metal Dichalcogenide Semiconductor. ACS Nano 2015, 9 , $647-655$.

(23) You, Y.; Zhang, X.-X.; Berkelbach, T. C.; Hybertsen, M. S.; Reichman, D. R.; Heinz, T. F. Observation of Biexcitons in Monolayer WSe2. Nat. Phys. 2015, 11, 477-481.

(24) Kim, J. C.; Wake, D. R.; Wolfe, J. P. Thermodynamics of Biexcitons in A GaAs Quantum Well. Phys. Rev. B 1994, 50, 15099-15107. 
(25) Sandorfy, C.; North-holland, E.; Yuan, Z.; Kardynal, B. E.; Stevenson, R. M.; Shields, A. J.; Lobo, C. J.; Cooper, K.; Beattie, N. S.; Ritchie, D. A.; Pepper, M. Electrically Driven SinglePhoton Source. Science 2002, 295, 102-105.

(26) Michler, P.; Kiraz, A.; Becher, C.; Schoenfeld, W. V. A Quantum Dot Single-Photon Turnstile Device. Science 2000, 290, 2282-2285.

(27) Chan, Y.; Steckel, J. S.; Snee, P. T.; Caruge, J. M.; Hodgkiss, J. M.; Nocera, D. G.; Bawendi, M. G. Blue Semiconductor Nanocrystal Laser. Appl. Phys. Lett. 2005, 86, 073102.

(28) Chan, Y.; Snee, P. T.; Caruge, J. M.; Yen, B. K.; Nair, G. P.; Nocera, D. G.; Bawendi, M. G. A Solvent-Stable Nanocrystal-Silica Composite Laser. J. Am. Chem. Soc. 2006, 128, 3146-3147.

(29) Cuthbert, J. D. Recombination Kinetics of Excitonic Molecules and Free Excitons in Intrinsic Silicon. Phys. Rev. B 1970, 1, 1552-1557.

(30) Gourley, P. L.; Wolfe, J. P. Thermodynamics of Excitonic Molecules in Silicon. Phys. Rev. B 1979, 20, 3319-3327.

(31) Gourley, P. L.; Wolfe, J. P. Experimental Determination of Equilibrium Constants for Excitonic Systems in Stressed, Ultrapure Silicon. Phys. Rev. B 1982, 25, 6338-6348.

(32) Rong, Y.; Fan, Y.; Leen Koh, A.; Robertson, A. W.; He, K.; Wang, S.; Tan, H.; Sinclair, R.; Warner, J. H. Controlling Sulphur Precursor Addition for Large Single Crystal Domains of WS2. Nanoscale 2014, 6, 12096-12103.

(33) Jo, S.; Ubrig, N.; Berger, H.; Kuzmenko, B. A.; Morpurgo, F. A. Mono- and Bilayer WS2 Light-Emitting Transistors. Nano Lett. 2014, 14, 2019-2025.

(34) Mak, K. F.; He, K.; Lee, C.; Lee, G. H.; Hone, J.; Heinz, T. F.; Shan, J. Tightly Bound Trions in Monolayer MoS2. Nat. Mater. 2013, 12, 207-211.

(35) Peimyoo, N.; Shang, J.; Yang, W.; Wang, Y.; Cong, C.; Yu, T. Thermal Conductivity Determination of Suspended Mono- and Bilayer WS2 by Raman Spectroscopy. Nano Res. 2014, $8,1210-1221$. 
(36) Berkdemir, A.; Gutiérrez, H. R.; Botello-Méndez, A. R.; Perea-López, N.; Elías, A. L.; Chia, C.-I.; Wang, B.; Crespi, V. H.; López-Urías, F.; Charlier, J.-C.; Terrones, H.; Terrones, M. Identification of Individual and Few Layers of WS2 Using Raman Spectroscopy. Sci. Rep. 2013, 3, 1755 .

(37) Plechinger, G.; Nagler, P.; Kraus, J.; Paradiso, N.; Strunk, C.; Schüller, C.; Korn, T. Identification of Excitons, Trions and Biexcitons in Single-Layer WS2. Phys. status solidi Rapid Res. Lett. 2015, 9, 457-461.

(38) Terrones, H.; Corro, E. Del; Feng, S.; Poumirol, J. M.; Rhodes, D.; Smirnov, D.; Pradhan, N. R.; Lin, Z.; Nguyen, M. A. T.; Elías, A. L.; Mallouk, T. E.; Balicas, L.; Pimenta, M. A.; Terrones, M. New First Order Raman-Active Modes in Few Layered Transition Metal Dichalcogenides. Sci. Rep. 2014, 4, 4215.

(39) Schmidt, T.; Lischka, K.; Zulehner, W. Excitation-Power Dependence of the near-Band-Edge Photoluminescence of Semiconductors. Phys. Rev. B 1992, 45, 8989-8994.

(40) Bansal, B.; Kadir, A.; Bhattacharya, A.; Moshchalkov, V. V. Photoluminescence from Localized States in Disordered Indium Nitride. Appl. Phys. Lett. 2008, 93, 021113.

(41) Chernikov, A.; Berkelbach, T. C.; Hill, H. M.; Rigosi, A.; Li, Y.; Aslan, O. B.; Reichman, D. R.; Hybertsen, M. S.; Heinz, T. F. Exciton Binding Energy and Nonhydrogenic Rydberg Series in Monolayer WS2. Phys. Rev. Lett. 2014, 113, 076802.

(42) Ye, Z.; Cao, T.; O’Brien, K.; Zhu, H.; Yin, X.; Wang, Y.; Louie, S. G.; Zhang, X. Probing Excitonic Dark States in Single-Layer Tungsten Disulfide. Nature 2014, 513, 214-218.

(43) Kleinman, D. A. Binding Energy of Biexcitons and Bound Excitons in Quantum Wells. Phys. Rev. $B$ 1983, 28, 871-879.

(44) Kiriya, D.; Tosun, M.; Zhao, P.; Kang, J. S.; Javey, A. Air-Stable Surface Charge Transfer Doping of $\mathrm{MoS}_{2}$ by Benzyl Viologen. J. Am. Chem. Soc. 2014, 136, 7853-7856.

(45) Conley, H. J.; Wang, B.; Ziegler, J. I.; Haglund, R. F.; Pantelides, S. T.; Bolotin, K. I. Bandgap Engineering of Strained Monolayer and Bilayer MoS2. Nano Lett. 2013, 13, 3626-3630. 
(46) Wang, S.; Wang, X.; Warner, J. H. All Chemical Vapor Deposition Growth of MoS2 : H-BN Vertical van Der Waals Heterostructures. ACS Nano 2015, 9, 5246-5254.

(47) Amin, B.; Kaloni, T. P.; Schwingenschlögl, U. Strain Engineering of WS2, WSe2, and WTe2. RSC Adv. 2014, 4, 34561.

(48) Rice, C.; Young, R. J.; Zan, R.; Bangert, U.; Wolverson, D.; Georgiou, T.; Jalil, R.;

Novoselov, K. S. Raman-Scattering Measurements and First-Principles Calculations of StrainInduced Phonon Shifts in Monolayer MoS2. Phys. Rev. B - Condens. Matter Mater. Phys. 2013, $87,1-5$.

(49) Desai, S. B.; Seol, G.; Kang, J. S.; Fang, H.; Battaglia, C.; Kapadia, R.; Ager, J. W.; Guo, J.; Javey, A. Strain-Induced Indirect to Direct Bandgap Transition in Multilayer WSe2. Nano Lett. 2014, 14, 4592-4597.

(50) Kresse, G.; Furthmüller, J. Efficient Iterative Schemes for Ab Initio Total-Energy Calculations Using a Plane-Wave Basis Set. Phys. Rev. B 1996, 54, 11169-11186.

(51) Perdew, J. P.; Burke, K.; Ernzerhof, M. Generalized Gradient Approximation Made Simple. Phys. Rev. Lett. 1996, 77, 3865-3868.

TOC graphic

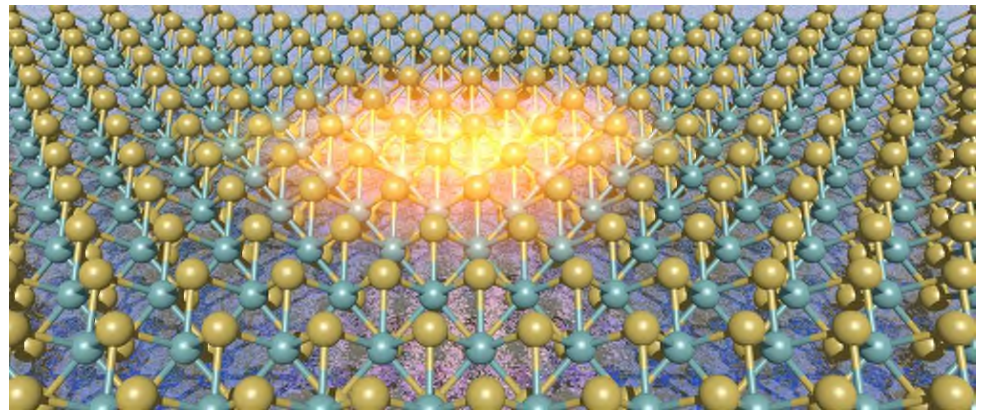

